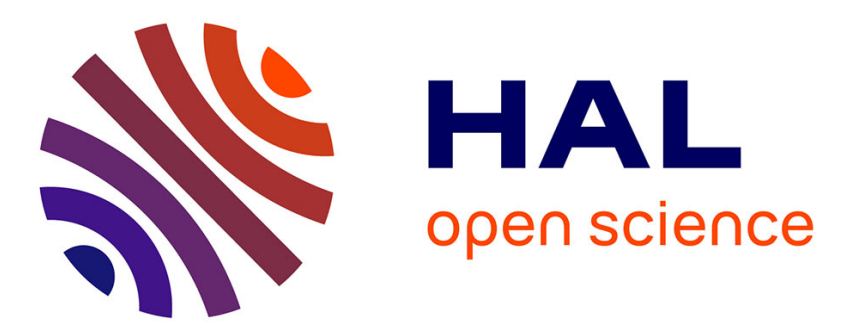

\title{
Noise characterization in millimeter sized micromanipulation systems.
}

Mokrane Boudaoud, Yassine Haddab, Yann Le Gorrec, Philippe Lutz

\section{To cite this version:}

Mokrane Boudaoud, Yassine Haddab, Yann Le Gorrec, Philippe Lutz. Noise characterization in millimeter sized micromanipulation systems.. Mechatronics, 2011, 21 (6), pp.1087-1097. 10.1016/j.mechatronics.2011.06.005 . hal-00672159

\section{HAL Id: hal-00672159 \\ https://hal.science/hal-00672159}

Submitted on 20 Feb 2012

HAL is a multi-disciplinary open access archive for the deposit and dissemination of scientific research documents, whether they are published or not. The documents may come from teaching and research institutions in France or abroad, or from public or private research centers.
L'archive ouverte pluridisciplinaire HAL, est destinée au dépôt et à la diffusion de documents scientifiques de niveau recherche, publiés ou non, émanant des établissements d'enseignement et de recherche français ou étrangers, des laboratoires publics ou privés. 


\title{
Noise characterization in millimeter sized micromanipulation systems.
}

\author{
M. Boudaoud, Y. Haddab, Y. Le Gorrec and P. Lutz \\ FEMTO-ST Institute, UMR CNRS 6174 - UFC / ENSMM / UTBM \\ Automatic Control and Micro-Mechatronic Systems Department \\ (AS2M). 24, rue Alain Savary 25000 Besançon FRANCE \\ (mokrane.boudaoud, yassine.haddab, legorrec, \\ philippe.lutz)@femto-st.fr
}

\begin{abstract}
:
Efficient and dexterous manipulation of very small (micrometer and millimeter sized) objects require the use of high precision micromanipulation systems. The accuracy of the positioning is nevertheless limited by the noise due to vibrations of the end effectors making it difficult to achieve precise micrometer and nanometer displacements to grip small objects. The purpose of this paper is to analyze the sources of noise and to take it into account in dynamic models of micromanipulation systems. Environmental noise is studied considering the following sources of noise: ground motion and acoustic noises. Each source of noise is characterized in different environmental conditions and a separate description of their effects is investigated on micromanipulation systems using millimeter sized cantilevers as end effectors. Then, using the finite difference method (FDM), a dynamic model taking into account studied noises is used. Ground motion is described as a disturbance transmitted by the clamping to the tip of the cantilever and acoustic noises as external uniform and orthogonal waves. For model validation, an experimental setup including cantilevers of different lengths is designed and a high resolution laser interferometer is used for vibration measurements. Results show that the model allows a physical interpretation about the sources of noise and vibrations in millimeter sized micromanipulation systems leading to new perspectives for high positioning accuracy in robotics micromanipulation through active noise control.
\end{abstract}

Keywords:

Environmental noise, micromanipulation systems, nanometer vibrations, characterization of noise sources, finite difference modeling.

\section{INTRODUCTION}

High precision micromanipulation tasks are required in a wide range of microrobotics applications, for instance microassembly [1], force sensing [2] and surgical operations [3]. In that sense, numerous micromanipulation systems (notably microgrippers) based on actuators with high positioning accuracy and good deformation/force ratio can be found in the literature [4] [5] [6]. The size of end effectors can vary from few micrometers [4] up to several tens of millimeters [7] [8]. While micrometric structures are suitable for high resolution positioning, millimeter ones have the advantage of offering large displacements. In most cases, noise and vibrations are the main factors reducing the performances of micromanipulation systems for both actuation [9] [10] [11] and sensing [12][13] leading to a low repeatability and a loss of accuracy. Moreover, when end effectors are used as sensors, vibrations are reflected in the sensor output as a measurement noise reducing significantly the sensing resolution [7] [14]. Undesired vibrations are partially caused by random motions of particles within materials known as thermal fluctuations leading to the thermal noise, and also by external perturbations com- ing from the surrounding environment reflecting the so called environmental noise. Thermal noise is predominant in micrometric structures such as AFM (Atomic Force Microscopy) cantilevers which are characterized with high resonance frequencies (in the order of tens of $\mathrm{KHz}$ ) [15] [16]. Environmental noise can include effects of external temperature, humidity, pressure, etc. and also relates to human activity, operating machines, etc. at a given location.

When a high positioning accuracy is needed, one has to deal with this noise. Numerous solutions are possible: designing environmental isolation platforms [17] [18], considering noise limitation during the design of micromanipulation systems by an appropriate choice of the resonance frequency of end effectors [19], or using appropriate controllers for noise rejection [20]. Multi-noise isolation platforms are generally very expansive and have a limited volume which can be a real problem if a micromanipulation station requires a large working space [21]. Moreover, vibration isolation tables commonly found in typical microrobotics laboratories allow efficient ground noise filetring but fail to filter acoustic noises. The perspective of our 
work is to provide a flexible solution, applicable in different environmental conditions for different micromanipulation tasks (microassembly [22], manipulation of biomaterials [12],) even if such operations require a large working space. Environmental noise is also usually not taken into account during microgrippers design. Then, the use of appropriate controllers for noise rejection is an interesting and low cost solution when considering a large kind of microgrippers. For this purpose, defining appropriate control strategies requires understanding the sources of noise and the manner in which they act on the process. For instance, in $H_{\infty}$ control strategy, a perturbation at the input (actuation) of a process must not be treated as the same way as the one disturbing the output (measurement). Moreover, the noise has to be considered differently according to the dimensions of the micromanipulation system.

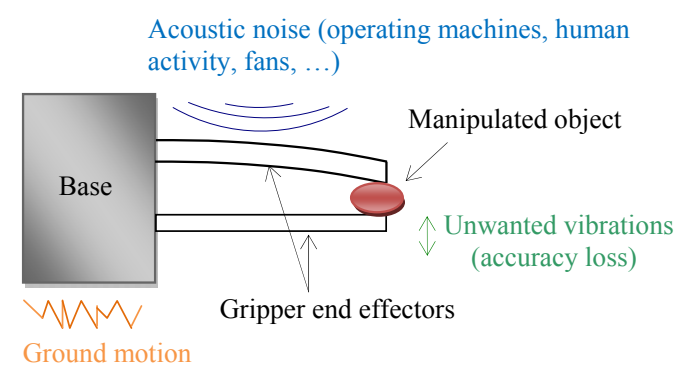

Fig. 1. Simplified scheme of a micromanipulation system using two cantilevers as end effectors under the influence of environmental noise.

Taking into account physical origins of noises is usually not addressed in conventional studies dealing with noise and vibration rejection in microrobotics [7] [20] [23] [24]. A better understanding about this will allow a more efficient controller design by an accurate modeling of the noisy process leading to the improvement of achievable closed loop performances. Indeed, this improvement depend on the accuracy of the noise characterization: magnitude, frequency shape, space location where the noise is applied on the system, etc.

The aim of this paper is then to perform a characterization of the main sources of noise disturbing micromanipulation systems and to include such noises into dynamic models of micromanipulators. The control part is not treated although it is the main perspective of the work. We have then focused our study on kind of micromanipulation systems using cantilevers as end effectors with lengths in the order of several tens of millimeters, such as those designed in [8] [25] [26]. Such systems are often characterized by low resonance frequencies (below $1 \mathrm{KHz}$ ) and are particularly sensitive to the environmental noise leading to significant vibrations around the resonance. As an experimental setup, cantilevers of different lengths clamped on a stiff support have been designed with respect to some criteria described in section 2.2. At these scales, thermal noise [27] leads to vibrations in the order of the Picometer which is insignificant comparing with ones produced by the environmental noise. For this reason, two main sources of noise are considered in this study: ground motion and acoustic noises. Ground motion is described as a disturbance transmitted by the clamping to the tip of the cantilever and acoustic noises as external uniform and orthogonal waves acting along the cantilever
(Fig. 1). The purpose is to identify noise sources from vibration measurements at the tip of a cantilever. To this end, a precise cantilever model is developed and used to reconstruct the noise source from the tip measurements.

This paper is organized as follows: first, ground motion and acoustic noises are described separately as perturbations in a dynamic modeling using the finite difference method (FDM). For an experimental characterization of the environmental noise, the instrumentation used in this study is described in the third section. Then, the environmental noise is characterized in a typical micromanipulation room and effect of each source of noise is evaluated on the experimental setup through vibration measurements at the tip of the cantilevers. In order to assess precisely about the single effect of acoustic noises, in the fifth section, experiments are conducted in an anechoic chamber where a loudspeaker is used to generate white acoustic noises from 0 to $70 d B$. The model allows the description of the source of noise through the power spectral density of the output. A good agreement is observed between simulations and experimental measurements allowing a fine characterization of the type of perturbation in control point of view. Conclusions and perspectives for further work are given in the last section.

\section{DYNAMIC MODELING OF A MICROMANIPULATION SYSTEM WITH ENVIRONMENTAL NOISE CONSIDERATION}

The Euler-Bernoulli equation is used for modeling one end effector of a micromanipulation system considering a cantilever type structure. The model is described with the hypothesis of small displacements. Then according to the finite difference method (FDM) for numerically solving partial differential equations (PDEs), the Euler-Bernoulli equation is translated into a state space representation.

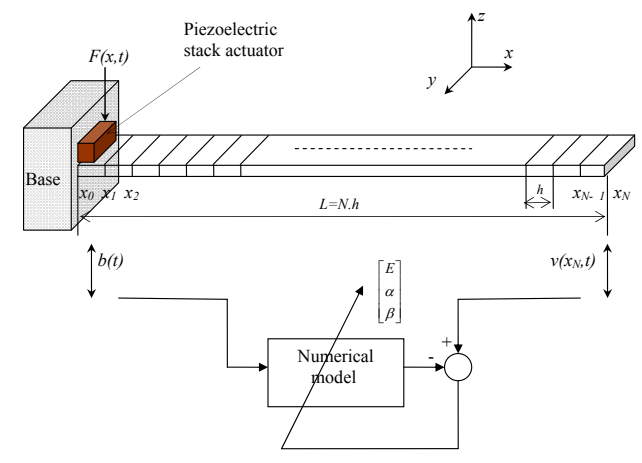

Fig. 2. Spatial discretization of a cantilever and optimal estimation of mechanical parameters.

\subsection{Finite difference formulation of the Euler-Bernoulli cantilever and state space representation}

Consider the PDE of Euler-Bernoulli describing the motion $v(x, t)$ of a beam with an applied force $F(x, t)$ per unit length:

$$
\text { E.I. } \frac{\partial^{4} v(x, t)}{\partial x^{4}}+\delta \cdot \frac{\partial v(x, t)}{\partial t}+\rho . S . \frac{\partial^{2} v(x, t)}{\partial t^{2}}=F(x, t)
$$

$E$ is the Young's modulus, $I$ is the area moment of inertia of the cross section, $\delta$ is the damping factor, $\rho$ is the mass 
density and $S$ defines the area of the cross section.

Partial derivatives in spatial dimension are approximated with the central finite difference scheme, using $N$ grid points (nodes) separated by a length of $h$ on the beam (Fig. 2). Later, $v\left(x_{n}\right)$ will designate the deflexion of the beam at a location $n \times h$.

According to the $4^{\text {th }}$ spatial derivative of equation (1), the finite difference approximation leads to:

$$
\begin{aligned}
\frac{\partial^{4} v\left(x_{n}, t\right)}{\partial x^{4}} & =\frac{v\left(x_{n+2}\right)-4 \cdot v\left(x_{n+1}\right)}{h^{4}} \\
& +\frac{6 \cdot v\left(x_{n}\right)-4 \cdot v\left(x_{n-1}\right)+v\left(x_{n-2}\right)}{h^{4}}
\end{aligned}
$$

For the case of a cantilever, the displacement and the slope must be zero at the fixed end, also the shear force and the bending moment must be zero at the free end (tip). Therefore, in mathematical terms this implies the following boundary conditions for equation (1):

$$
\left\{\begin{array} { l } 
{ v ( x _ { 0 } ) = 0 } \\
{ v ^ { \prime } ( x _ { 0 } ) = 0 }
\end{array} \quad \left\{\begin{array}{l}
v^{\prime \prime}\left(x_{N}\right)=0 \\
v^{\prime \prime \prime}\left(x_{N}\right)=0
\end{array}\right.\right.
$$

Then:

$$
\left\{\begin{array} { l } 
{ v ( x _ { 0 } ) = 0 } \\
{ v ( x _ { 1 } ) = v ( x _ { - 1 } ) }
\end{array} \left\{\begin{array}{l}
v\left(x_{N-1}\right)=2 . v\left(x_{N}\right)-v\left(x_{N+1}\right) \\
v\left(x_{N-2}\right)=4 . v\left(x_{N}\right)-4 . v\left(x_{N+1}\right)+v\left(x_{N+2}\right)
\end{array}\right.\right.
$$

Using equation (2) and the boundary conditions, a linear relationship between the vector $V=\left[v\left(x_{0}\right) \cdots \cdots v\left(x_{N}\right)\right]^{T}$ and its fourth spatial derivative is expressed through the matrix [derive4]:

$$
\text { [derive }]=\left(\begin{array}{cccccccc}
0 & -8 & 1 & 0 & & \cdots & & 0 \\
0 & 7 & -4 & 1 & 0 & \cdots & & 0 \\
0 & -4 & 6 & -4 & 1 & 0 & \cdots & 0 \\
0 & \ddots & \ddots & \ddots & \ddots & \ddots & \ddots & \vdots \\
\vdots & 0 & 1 & -4 & 6 & -4 & 1 & 0 \\
0 & \cdots & 0 & 1 & -4 & 6 & -4 & 1 \\
0 & & \cdots & 0 & 1 & -4 & 5 & -2 \\
0 & & \cdots & & 0 & 2 & -4 & 2
\end{array}\right)
$$

The partial differential equation (1) is then converted into matrix equation by computing the matrices $M, Q$, $K$ and $M_{f}$, respectively the mass, damping, stiffness and load matrices:

$$
M . \ddot{V}+Q \cdot \dot{V}+K \cdot V=M_{f} \cdot F
$$

$$
\begin{aligned}
& M=\rho . S \cdot I_{N}, K=E \cdot I \cdot \frac{1}{h^{4}}[\text { derive } 4] \text { and } Q=\delta . I_{N} \\
& I_{N} \in \Re^{N \times N} \text { is the identity matrix }
\end{aligned}
$$

The effect of the environmental noise is described through the matrix $M_{f}$. Indeed this last indicates the manner in which external disturbances are acting on the structure.
In the case of our study, we have chosen to divide $M_{f}$ into two matrices: the first one $M_{f b} \in \Re^{N \times 1}$ relates to the motion of the base, and the second one $M_{f c} \in \Re^{N \times 1}$ to the acoustic noise.

For ground motion expressed by the variable $b(t)$, the previous boundary conditions are changed for the clamped part and are defined as:

$$
\left\{\begin{array} { l } 
{ v ( x _ { 0 } , t ) = b ( t ) } \\
{ v ^ { \prime } ( x _ { 0 } ) = 0 }
\end{array} \Rightarrow \left\{\begin{array}{l}
v\left(x_{0}, t\right)=b(t) \\
v\left(x_{1}\right)=v\left(x_{-1}\right)
\end{array}\right.\right.
$$

In terms of force, with respect to the FDM, the force produced by the ground motion on the base can be expressed as follow:

$$
F_{b}(t)=\frac{E . I}{h^{4}} \cdot b(t)
$$

The load matrix $M_{f b}$ is then obtained as:

$$
M_{f b}=\left[\begin{array}{llllll}
-6 & 4 & -1 & 0 & \cdots & 0
\end{array}\right]^{T}
$$

This matrix allows the description of a displacement $v\left(x_{i}, t\right)$ at any space location of the cantilever $(1<i<N)$ starting from the displacement $v\left(x_{0}\right)=b(t)$.

On the other hand, only the part of acoustic noises which are acting in the direction of flexion ( $\mathrm{z}$ axis) are considered. Indeed, in this direction, the effect of acoustic noise is predominant on the cantilever. The model is based on the hypothesis on plane acoustic noises. In this case, we assume pinpoint forces $F_{c}(t)$ acting at each node of the discretized scheme. This consideration allows defining:

$$
M_{f c}=\left[\begin{array}{lllll}
0 & 1 & 1 & \cdots & 1
\end{array}\right]^{T}
$$

In this matrix, the term 0 defines that the base in not affected by the acoustic noise. The limit of this consideration is investigated in section 5 , where effects of acoustic noises are analyzed in an anechoic chamber.

From equation (3), a state space model is derived considering as temporal states: the displacement and the velocity of each node of the discretized cantilever:

$$
\left\{\begin{array}{l}
\dot{X}=A \cdot X+B \cdot F \\
v\left(x_{N}\right)=C \cdot X
\end{array}\right.
$$

$$
\begin{aligned}
& X=\left[\begin{array}{ll}
V & \dot{V}
\end{array}\right]^{T}, F=\left[\begin{array}{ll}
F_{b} & F_{c}
\end{array}\right]^{T}, \\
& A=\left(\begin{array}{cc}
0_{N \times N} & I_{N} \\
-M^{-1} . K & -M^{-1} \cdot Q
\end{array}\right) \\
& B=\left(\begin{array}{cc}
0_{N \times 1} & 0_{N \times 1} \\
M^{-1} \cdot M_{f b} & M^{-1} \cdot M_{f c}
\end{array}\right), C=\left(\begin{array}{lll}
0_{1 \times(N-1)} & 1 & 0_{1 \times N}
\end{array}\right) \\
& A \in \Re^{2 N \times 2 N}, B \in \Re^{2 N \times 2} \text { and } C \in \Re^{1 \times 2 N} .
\end{aligned}
$$

This kind of representation is helpful for an easy computation of the model in the Matlab/Simulink software allowing the description of the dynamic response of the modeled structure under several inputs (environmental 
noise). Moreover, this representation is often used in control systems. Then, according to the model, tip vibrations $v\left(x_{N}\right)$ of an end effector can be simulated using experimental measurements of ground motion and acoustic noises.

\subsection{Description of the experimental setup}

Four cantilevers of different lengths (TABLE 1) have been designed starting from a unique bulk of aluminum. A wire processing allowed machining into the bulk in order to obtain cantilevers of different lengths with the same cross section area (the base is the non machined part) (Fig. 3).

The design of the cantilevers followed criteria in terms of dimension, stiffness and resonance frequency aiming at being as close as possible to commonly used micromanipulation systems with millimeter sized dimensions. Typical dimensions of such systems are $(40 \mathrm{~mm} \times 10 \mathrm{~mm} \times 0.2 \mathrm{~mm})$ [8], $(15 \mathrm{~mm} \times 2 \mathrm{~mm} \times 0.3 \mathrm{~mm})$ [25] and $(23.8 \mathrm{~mm} \times 3.4 \mathrm{~mm} \times$ $0.16 \mathrm{~mm})[26]$. The stiffness of micromanipulation systems can vary from tens to hundreds $N / m$, such as the one studied in a previous work [28]. Moreover the resonance frequency of millimeter sized micromanipulation systems is most of time below $1 \mathrm{KHz}$. For instance, in references [7], [25] and [29], the resonance of the microgrippers are $700 \mathrm{~Hz}, 600 \mathrm{~Hz}$ and $200 \mathrm{~Hz}$ respectively.

Millimeter sized micromanipulation systems are often used to perform handling operations on objects with sizes ranging from several micrometers to few millimeters. The load handling capability of the micromanipulation system depends on the achievable displacement at the free end of the arm. The gripping force can then be deduced from the stiffness of the arm.
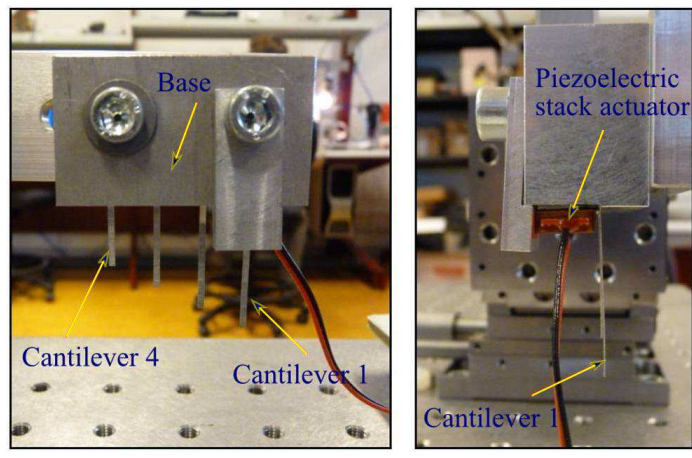

Fig. 3. Cantilevers of different lengths used as an experimental setup.

Effect of environmental noise will be investigated on the four cantilevers. Nevertheless, the modeling approach described above will be affined according only to the dynamic of the cantilever 1 (the same work can be done on the other cantilevers).

For this purpose, a piezoelectric stack actuator ( $\mathrm{P}$ 249.20, Physik Instrument $\mathrm{GmbH}$ ) with $20 \mathrm{KHz}$ resonance frequency has been fixed near the clamped part of the cantilever 1 for a mechanical characterization of the structure (derivation of the damping and the Young's modulus) through base excitations. Experimental measurements showed that, for small displacements (less than $50 \mathrm{~nm}$ ), the actuator obeys to a linear supply volt- age/displacement characteristic with a sensitivity equal to $K_{a c t}=1.6 \mathrm{~nm} /$ Volts .

Table 1. Dimensions, stiffness and resonance frequency of designed cantilevers

\begin{tabular}{lllll}
\hline & cantilever1 & cantilever2 & cantilever3 & cantilever4 \\
\hline Length & $30 \mathrm{~mm}$ & $25 \mathrm{~mm}$ & $20 \mathrm{~mm}$ & $15 \mathrm{~mm}$ \\
\hline Width & $1.5 \mathrm{~mm}$ & $1.5 \mathrm{~mm}$ & $1.5 \mathrm{~mm}$ & $1.5 \mathrm{~mm}$ \\
\hline Thickness & $0.22 \mathrm{~mm}$ & $0.22 \mathrm{~mm}$ & $0.22 \mathrm{~mm}$ & $0.22 \mathrm{~mm}$ \\
\hline Stiffness & $9.9 \mathrm{~N} / \mathrm{m}$ & $17.12 \mathrm{~N} / \mathrm{m}$ & $33.44 \mathrm{~N} / \mathrm{m}$ & $79.26 \mathrm{~N} / \mathrm{m}$ \\
\hline Resonance & $173 \mathrm{~Hz}$ & $245 \mathrm{~Hz}$ & $375 \mathrm{~Hz}$ & $644 \mathrm{~Hz}$ \\
\hline
\end{tabular}

\subsection{Validation of the finite difference model}

In response to a $1 V$ step excitation at the input of the actuator, tip vibrations of the cantilever 1 have been recorded using a high resolution $(0.01 \mathrm{~nm})$ laser interferometer sensor (SP-120 SIOS Mebtechnik GmbH).

For the estimation of the damping, according to $N=$ 512 grid points in the discretized scheme, the damping matrix has been computed using Lord Rayleigh's hypothesis [30], for which:

$$
Q=\alpha \cdot K+\beta \cdot M
$$

Weighting parameters $\alpha$ and $\beta$ and the Young's modulus $E$ have been optimized (Fig. 2) using the recorded step response of the cantilever 1 and a least squares identification method (Levenberg-Marquardt algorithm). The Young's modulus has been optimized in order to capture the resonance frequency as close as the real one, and the optimization of the damping is conducted in order to get in the model a fine description of the effect of environmental noise around the resonance. During the optimization procedure, inputs of the model have been: $b=1.6 \mathrm{~nm}$ and $F_{c}=0$.

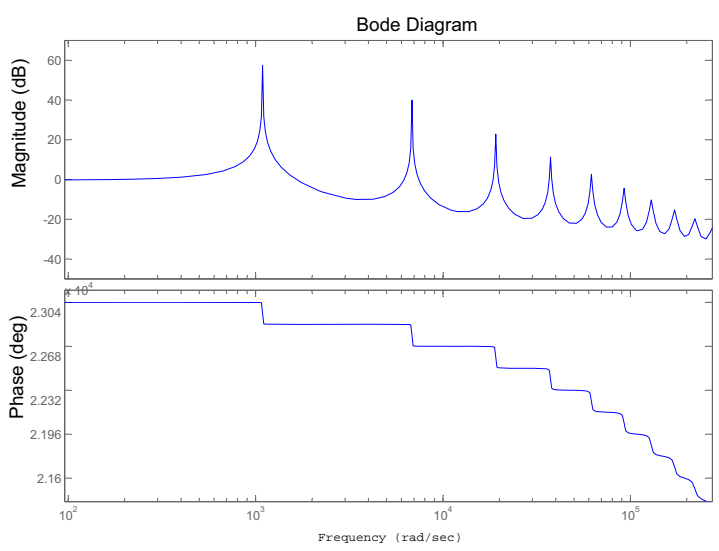

Fig. 4. Bode diagram of the model between input (base excitation) and output (deflexion of the free end) simulated by Matlab.

Starting from initial values of the parameters to be optimized such as: $E=67 G P a, \alpha=1.10^{-9}$ and $\beta=1$, optimal parameters leading to a minimum value of error between the output of the model and the experimental step response have been defined as: $E=67.15 \mathrm{GPa}$, $\alpha=2.31 .10^{-7}$ and $\beta=1.3572$. 
Dimensions of the cantilever used in the model are those defined in (TABLE 1, cantilever 1 ) and the mass density has been fixed at $\rho=2900 \mathrm{Kg} / \mathrm{m}^{2}$. Bode diagrams of the numerical model with base excitation and uniform and orthogonal acoustic waves are presented in (Fig. 4) and (Fig. 5) respectively.

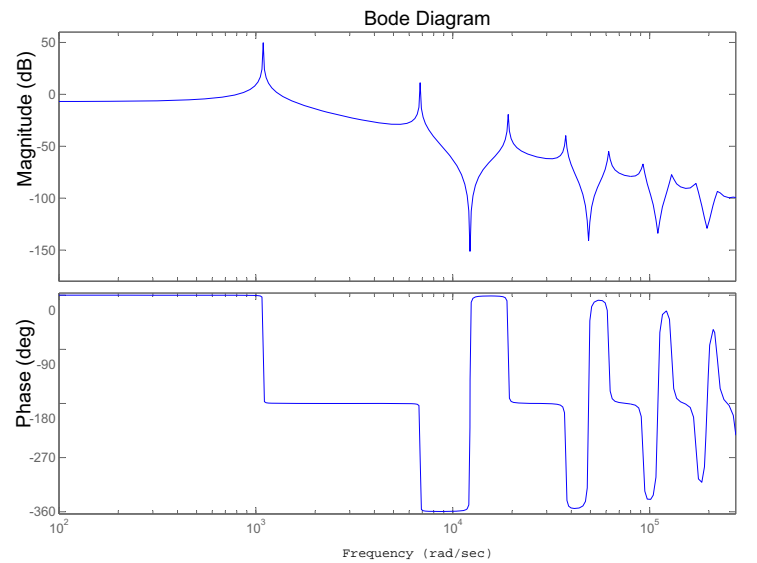

Fig. 5. Bode diagram of the model between input (uniform and orthogonal acoustic waves) and output (deflexion of the free end) simulated by Matlab.

In (Fig. 6) and (Fig. 7), it is possible to observe the validity of the numerical model according to a sinusoidal base excitation. A sinusoidal voltage with a magnitude of $31.25 \mathrm{~V}$ has been applied to the piezoelectric stack actuator at the first resonance frequency of the cantilever 1. For the simulation, model inputs have been: $b(t)=$ $50 . \sin (2 . \pi .173 . t) n m$ and $F_{c}=0$.

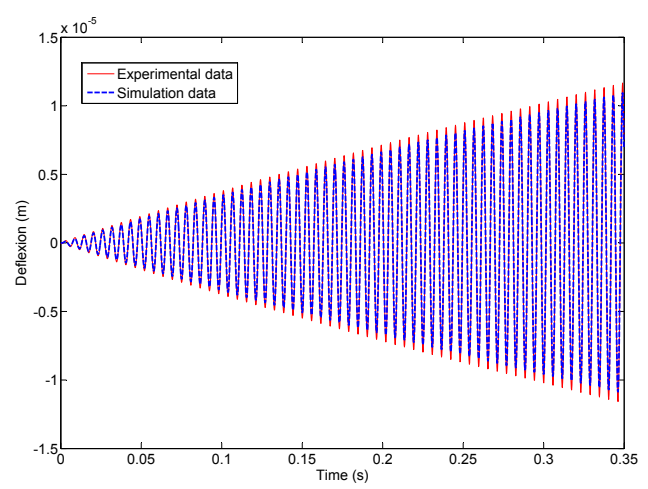

Fig. 6. Dynamic response of the cantilever 1 for sinusoidal base excitation at the first resonance frequency (time domain).

\section{INSTRUMENTATION FOR NOISE CHARACTERIZATION}

Considering that an important part of ground motion is transmitted to the base of the cantilever, the characterization of this source of noise is performed by vibration measurements at the base. This is conducted using the laser interferometer sensor (SP-120 SIOS Mebtechnik $\mathrm{GmbH}$ ) which covers a frequency range from few hertz up to $1 M H z$. The distance maintained between the laser interferometer sensor and the system (i.e. base or the free end of the cantilevers) is equal to $50 \mathrm{~mm}$.
Moreover, acoustic noises acting in the direction of flexion are recorded using a dedicated microphone located close to the cantilevers (Fig. 8). Acoustic noises can be produced by human activity (speech), operating machines in a given location or by a loudspeaker. For such mea-

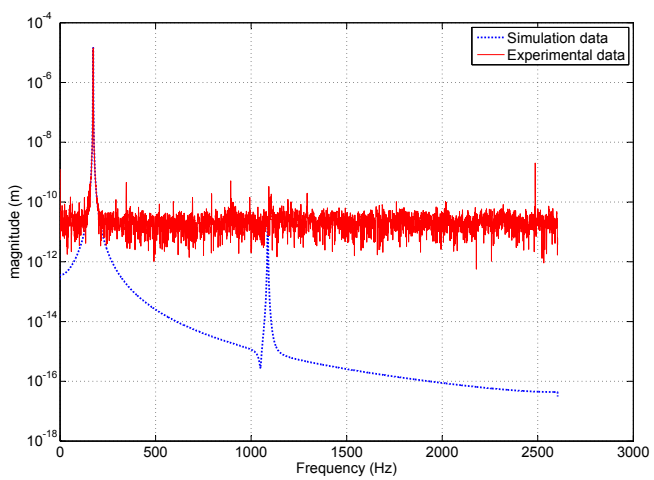

Fig. 7. Dynamic response of the cantilever 1 for sinusoidal base excitation at the first resonance frequency (frequency domain).

surements, a microphone $(1 / 2 " 50 \mathrm{mV} / \mathrm{Pa})$ from (01dBmetravib) has been used. This microphone allows acoustic noise measurements from $3.15 \mathrm{~Hz}$ up to $20 \mathrm{KHz}$. For the characterization of acoustic noises, a loudspeaker (LD 130 CR08 - ATOHM) with $44 \mathrm{~Hz}$ resonance frequency is used in section 5. This loudspeaker has a flat acoustic pressure/frequency characteristic from $100 \mathrm{~Hz}$ up to $1 \mathrm{KHz}$ allowing the generation of white acoustic noises in this frequency bandwidth. Finally, a vibration isolation table (kinetic systems, vibraplane, model no 9101-21-46) is used for ground motion attenuation.

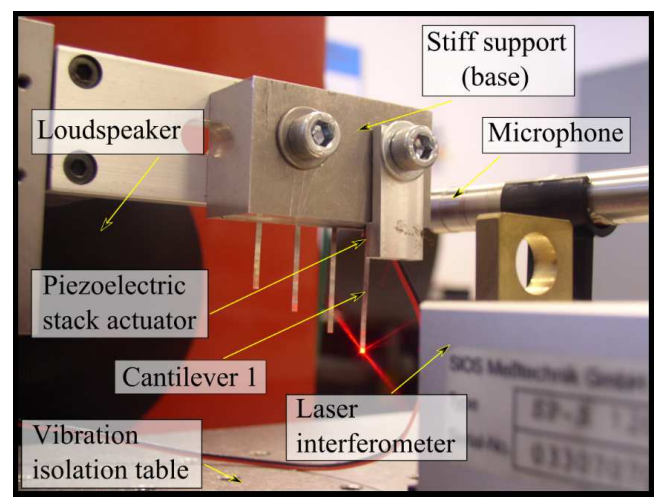

Fig. 8. Instrumentation for noise characterization.

Ground motion and acoustic noises recorded in a given environment can be set in the model inputs. Output of the model can be compared with experimental vibration measurements at the tip of the cantilever for the characterization of the sources of noise (Fig. 9)(Fig. 10).

Using this approach, an active control strategy can be defined according whether the predominant origin of tip vibrations is due to acoustic or ground motion.

For the case of micromanipulation systems, environmental noise within a typical micromanipulation room has to be characterized and effects of each source of noise on the experimental setup have to be assessed. Then according to 


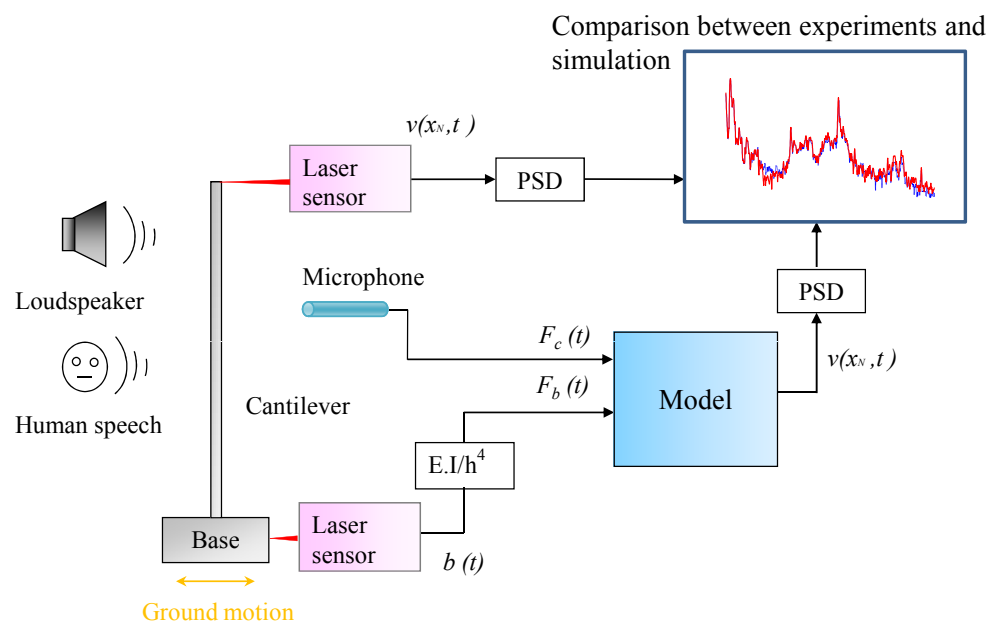

Fig. 10. Characterization of origin of noises using experimental measurements and the numeric model.

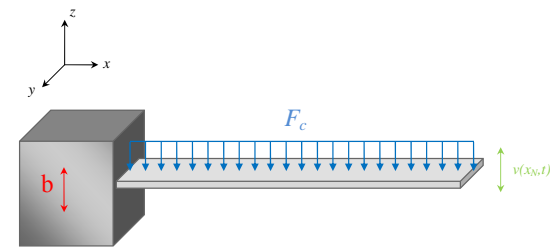

Fig. 9. Cantilever subject to acoustic noise $F_{c}$ and ground motion $b$ used as model inputs

experimental vibrations measurements at the cantilevers tip, origin of noise must be defined.

\section{ENVIRONMENTAL NOISE WITHIN A MICROMANIPULATION ROOM}

\subsection{Ground motion and acoustic noise measurements}

Ground motion is often characterized by a complex spectrum consisting of fast and slow motions [31] [32]. While slow motions relate on the own activity of the earth which is concentrated below few $H z$, it is the cultural noise with frequencies higher than a few hertz that has the potential of affecting flexible structures and especially near their resonance frequencies by acting on clamped parts. Cultural noise relating mainly on operating machines has generally a significant energy from a few $\mathrm{Hz}$ up to a few hundred $\mathrm{Hz}$ [33] and its frequency spectrum can vary from one location to another.

Ground motion has been measured in a typical micromanipulation room (Automatic Control and MicroMechatronic Systems Department, FEMTO-ST institute) during the day when all usual sources of noise are present (running computers, electronic amplifiers, experimental setup for noise analysis, etc.) (Fig. 11)

The laser interferometer sensor has been used in order to perform horizontal vibration measurements on the base during one hour. In order to assess the effectiveness of the vibration isolation table, measurements have been performed on the vibration isolation table and out of this last using a simple table located on the floor. On the other hand, acoustic noises have been measured during the day in two different environmental conditions: low

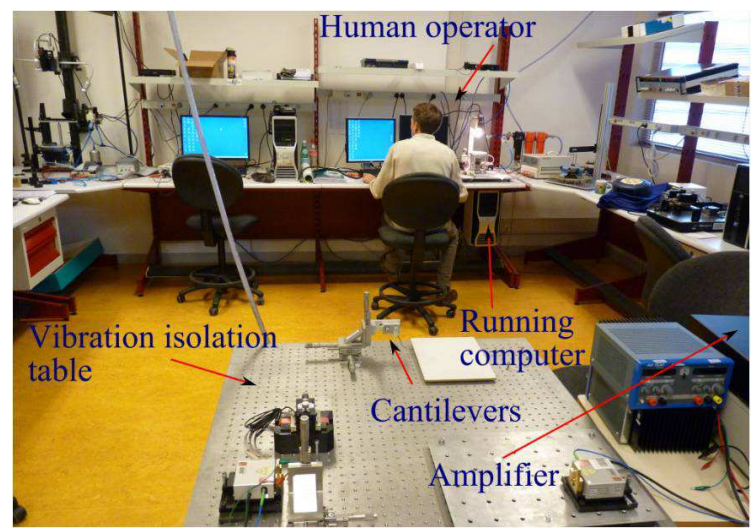

Fig. 11. Typical micromanipulation room (Automatic Control and Micro-Mechatronic Systems Department, FEMTO-ST institute)

and high human activity. Low human activity refers to the environmental condition corresponding to about $30 \mathrm{~dB}$ acoustic noises which are usually produced by one to three people. High human activity refers to noises of more than $45 d B$ which corresponds to ten to twelve people talking in the laboratory. Each acoustic noise measurement is performed close to the cantilevers during 60seconds.

Power and amplitude spectral densities (PSDs and ASDs) of ground motion and acoustic noise respectively have been computed starting from measurements data and averaged 128times according to the Welch method using the MATLAB software. Results are shown in (Fig. 12) and (Fig. 13) respectively.

The effectiveness of the vibration isolation table for ground motion reduction is clearly observed (Fig. 12). However, out of this table, the PSD of ground motion generally decreases with the increasing frequency and vanishes beyond $350 \mathrm{~Hz}$ (below the resolution of the laser interferometer). The measured ground motion in this case is mainly due to the cultural noise. Moreover human activity can produce an important increase of acoustic noises (Fig. 13). Then, acoustic noises even produced by humans must be also considered as a significant source of noise. 
Table 2. r.m.s. vibration at the free end of the cantilevers subject to the environmental noise.

\begin{tabular}{lllllll}
\hline & $\begin{array}{l}\text { vibration } \\
\text { isolation }\end{array}$ & $\begin{array}{l}\text { human } \\
\text { activity }\end{array}$ & cantilever 1 & cantilever 2 & cantilever 3 & cantilever 4 \\
\hline condition 1 & off & low & $112.8 \mathrm{~nm}$ & $19.1 \mathrm{~nm}$ & $5 \mathrm{~nm}$ & $0.4 \mathrm{~nm}$ \\
\hline condition 2 & on & high & $123.8 \mathrm{~nm}$ & $20 \mathrm{~nm}$ & $6 \mathrm{~nm}$ & $0.5 \mathrm{~nm}$ \\
\hline condition 3 & on & low & $7.2 \mathrm{~nm}$ & $0.9 \mathrm{~nm}$ & $0.45 \mathrm{~nm}$ & $0.1 \mathrm{~nm}$ \\
\hline
\end{tabular}

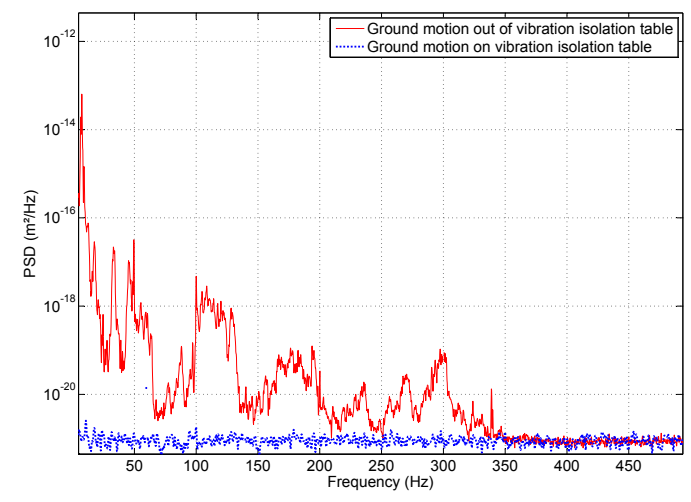

Fig. 12. Power spectral density of ground motion within the micromanipulation room.

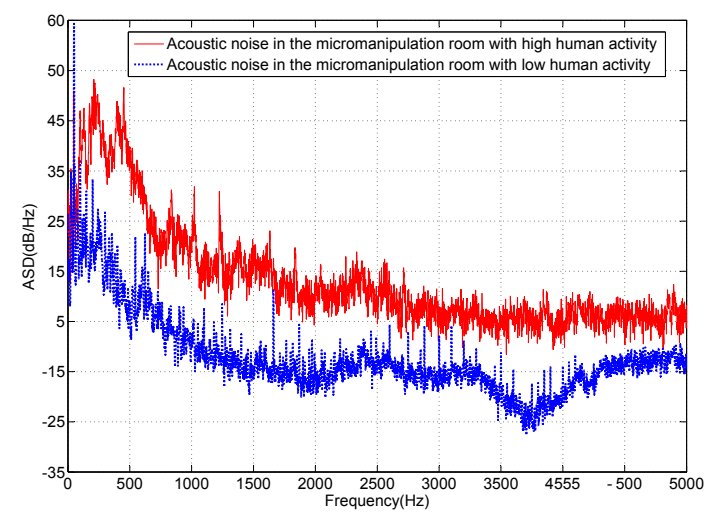

Fig. 13. Amplitude spectral density of acoustic noise within the micromanipulation room.

4.2 Effects of acoustic noise and ground motion on the cantilevers within the micromanipulation room

In order to assess separately about the effect of ground motion and acoustic noise on the designed cantilevers, vibration measurements at the tip of each cantilever are performed in different environmental conditions. The effect of environmental noise is evaluated basing on the root mean square (r.m.s.) level of vibrations at the tip of each cantilever.

Effect of ground motion is studied out of the vibration isolation table in low human activity (condition 1), and those of acoustic noises are evaluated on the vibration isolation table in high human activity (condition 2). Moreover, vibration measurements at the tip of the cantilevers are also performed on the vibration isolation table with low human activity (condition 3) where both sources of noise are reduced. During all experiments, temperature and pressure parameters have remained constant and have been equal to about $22^{\circ} \mathrm{C}$ and $98015 \mathrm{~Pa}$ respectively.

For each measurement, PSDs are computed as shown for instance in (Fig. 14) (Fig. 15) for conditions 1 and 3

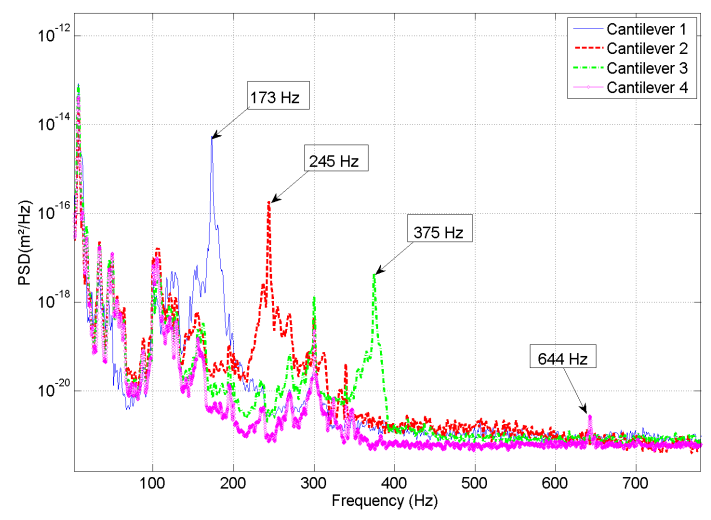

Fig. 14. Power spectral density of cantilevers tip vibrations out of the vibration isolation table within the micromanipulation room (low human activity)- Condition 1.

respectively. The r.m.s. values of vibrations are derived according to:

$$
r . m . s\left(f_{1}, f_{2}\right)=\sqrt{\sum_{f_{1}}^{f_{2}} P S D(f) . \Delta f}
$$

With $\Delta f=f_{2}-f_{1}=150 \mathrm{~Hz}$ is the frequency bandwidth where the PSD is the most significant (around the first flexural resonance).

By referring to TABLE 2, in a typical micromanipulation room, due to the environmental noise, r.m.s. vibrations are in the nanometer range. This may be a severe limitation if a nanometer accuracy is needed in a micromanipulation task. For instance, in (Fig. 16), due to the only human activity, it is possible to observe that performing tip displacement of the cantilever 1 with amplitudes lower than $100 \mathrm{~nm}$ is highly difficult in condition 2, while this can be easier in condition 3 . In the microworld, positioning accuracy depends on the size of the manipulated object. If the required accuracy is 100 times smaller than the size of the object, the manipulation of a red blood cell of $10 \mu \mathrm{m}$ diameter cannot be achieved with the desired performances in the environmental conditions 1 and 2 .

Moreover, r.m.s. of vibrations in conditions 1 and 2 are close to each other for all cantilevers, although they are produced by different sources of noise and the manner in which they act on the system is different. This is a real problem when considering a strategy for active noise rejection.

Acoustic noises proved that they have an important contribution on the vibrations of the cantilevers although produced at low acoustic levels (Fig. 13). This noise has the most important effect when it acts as an orthogonal wave in the direction of flexion of the cantilever. Moreover, 
for the modeling point of view, it is important to know whether acoustic noises generate also vibrations on the base in which case they have to be considered as coming from the ground motion.

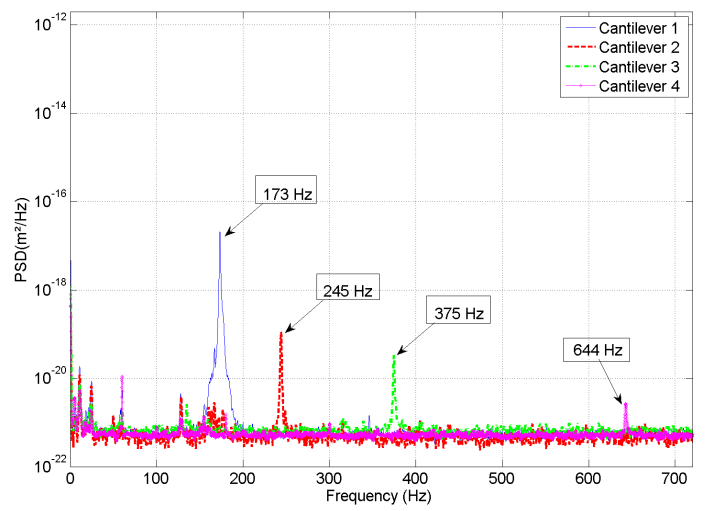

Fig. 15. Power spectral density of cantilevers tip vibrations on the vibration isolation table within the micromanipulation room (low human activity)- Condition 3.

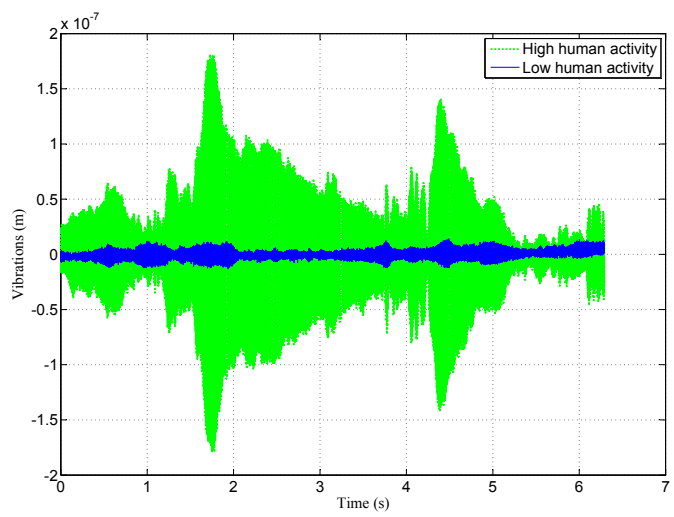

Fig. 16. Increase of the vibrations at the tip of cantilever 1 by increasing human activity in the micromanipulation room (on vibration isolation table)- exctraction of a measurement on 6.3 seconds duration.

Then, in order to assess about the effect of unidirectional acoustic noises, and to analyze the sensitivity of the base to acoustic pressures, experiments have been performed in an anechoic chamber.

\section{ACOUSTIC NOISE STUDY IN AN ANECHOIC CHAMBER}

The single effect of unidirectional acoustic noises is inspected in an anechoic chamber. This room is generally designed to reduce reflections of sound and electromagnetic waves and is insulated from exterior sources of noise. The experimental setup including the cantilevers, the laser interferometer, the microphone and the loudspeaker has been moved to the anechoic chamber of the FEMTO-ST institute (Fig. 17).

In this chamber, the walls and the ground are covered with a rubberized material foam such as reflections of sound and electromagnetic waves are reduced and insulated from exterior sources of noise. Moreover the transmission of vibrations from the ground is also greatly minimized.
The loudspeaker has been placed at 1 meter distance from the experimental setup such as acoustic noises are considered as uniform and orthogonal waves along the cantilevers.

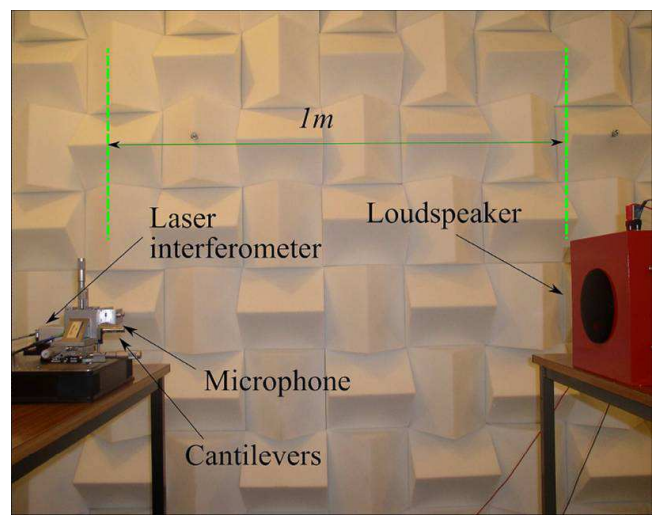

Fig. 17. Experimental setup within the anechoic chamber for the analysis of the effect of acoustic noise.

To assess the level of environmental noise in this chamber, vibrations measurements have been performed on the base and acoustic noises have been recorded when the loudspeaker was generating no sound. PSDs and ASDs of ground motion and acoustic noises are then computed and compared with ones found in the micromanipulation room (Fig. 18) (Fig. 19). The high environmental quality of such chambers is then observed.

For the characterization of the noise, the loudspeaker generated white acoustic noises from $100 \mathrm{~Hz}$ to $1 \mathrm{KHz}$, at different acoustic levels: $10 \mathrm{~dB}$ until $70 \mathrm{~dB}$.

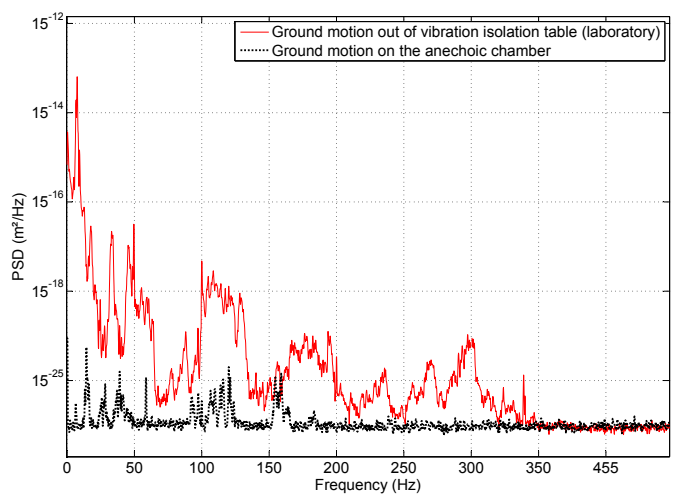

Fig. 18. Power spectral density of ground motion within the anechoic chamber.

For each generated noise, vibration measurements are performed at both the base and the free end of each cantilever. Then r.m.s. vibrations are derived from $P S D s$ in $150 \mathrm{~Hz}$ bandwidth around the resonance of the cantilevers such as performed previously. According to recorded data, the evolution of the r.m.s. vibrations at both the base and the free end of each cantilever has been assessed versus acoustic levels.

As shown in (Fig. 20), the r.m.s. vibration at the free part of the cantilever 1 increases significantly with the level of acoustic noises while the increase of the r.m.s. vibration at the base is much slower. However, from $30 \mathrm{~dB}$, significant vibrations at the base $(>1 \mathrm{~nm})$ 
are observed. These vibrations can reach $92 \mathrm{~nm}$ for $70 \mathrm{~dB}$ acoustic pressure. This demonstrates that acoustic noises have also an important effect on the base. This last has a contribution on the cantilevers tip vibrations. Considering acoustic noises as a perturbation acting only along the cantilevers is then not sufficient in the modeling, the effect of acoustic noises on the base must be also taken into account and especially when exceeding $30 \mathrm{~dB}$.

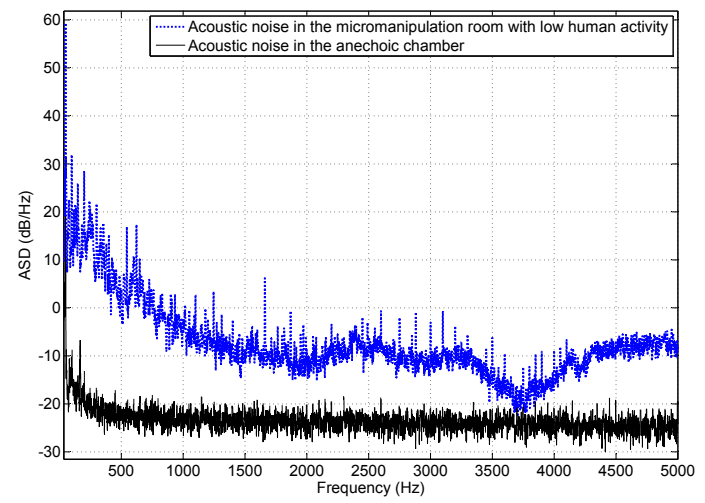

Fig. 19. Amplitude spectral density of acoustic noise within the anechoic chamber.

An accurate characterization of the sources of noise by modeling requires defining the sensitivity of the clamping (base) to acoustic noise. In this case all residual vibrations located at the base have to be added to the model although a high isolation of the cantilever from the ground is guaranteed (case of a vibration isolation table).

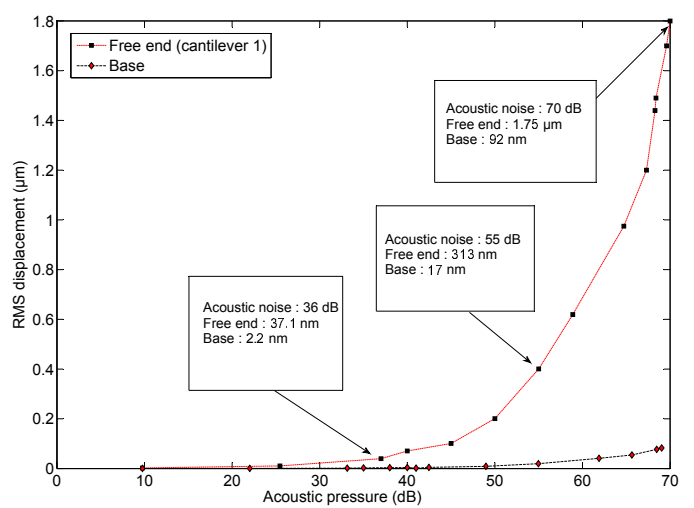

Fig. 20. r.m.s. vibration levels of cantilever 1 (tip and base)versus acoustic noise levels.

From measurements of (Fig. 20), based on Lagrange polynomials, an interpolation of the curve describing r.m.s $\left(v\left(x_{N}\right)\right.$ ) (tip vibrations) and r.m.s(b) (base vibrations) in terms of acoustic pressures expressed in Pascal has been performed. Then considering acoustic pressures $P_{d B}$ in $d B$, led us obtaining for the cantilever 1:

$$
\begin{aligned}
& r . m . s\left(v\left(x_{N}\right)\right)=\left(27.652 \times 20 \times 10^{-6} \times 10^{\left(P_{d B} / 20\right)}\right)+0.0021963[\mu m] \\
& r . m . s(b)=\left(1.4554 \times 20 \times 10^{-6} \times 10^{\left(P_{d B} / 20\right)}\right)+0.0003941[\mu m]
\end{aligned}
$$

For $45 d B$ acoustic noises, r.m.s. vibrations at the tip of the cantilever 1 are about $100.5 \mathrm{~nm}$. This is close to the level of vibrations found in condition 2 in high human activity (see TABLE 2).
Moreover, for $20 \mathrm{~dB}$, tip vibrations are $7.7 \mathrm{~nm}$, letting appear that in the condition 3 , the vibrations measured at the tip of the cantilever 1 are mostly due to acoustic effect.

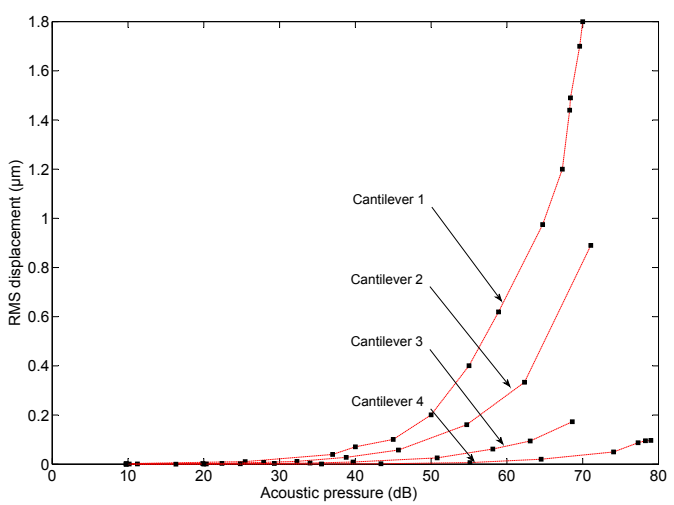

Fig. 21. r.m.s. vibration levels of all cantilevers versus acoustic noise levels.

\section{NUMERICAL SIMULATION OF THE EFFECT OF GROUND MOTION AND ACOUSTIC NOISE ON THE CANTILEVER 1}

In this section, experimental measurements of environmental noise are set in the model inputs. We have then considered two environmental conditions for the validation of the model. The first one is condition 1 described in section 4.2 , where ground motion is predominant. The second one relates to the experiments performed in the anechoic chamber when the loudspeaker was generating $45 \mathrm{db}$ (equivalent to the condition 2 - high human activity).

For the first case, experimental measurements of the ground motion have been set in the variable $b(t)$, and $F_{c}$ has been set at zero considering it is not significant in low human activity. Results are shown in (Fig. 22) for the cantilever 1 and in (Fig. 23) for the cantilever 2 as a comparative. A good agreement is observed letting appear that the model is able to take into account a perturbation whose origin is the ground motion.

For the second case, model inputs have been: the motion of the base recorded in the given condition and uniform and orthogonal acoustic waves, such as for each node of the numerical scheme, the same force per unit length $F_{c}=5.4 \mu \mathrm{N} / \mathrm{m}$ has been used which is equal to the acoustic noise expressed in $N / \mathrm{m}^{2}$ times the width of the cantilever. PSDs of the model output and the one of the experimental measurements are presented in (Fig. 24) for the cantilever 1 . The good agreement of the two signals has been obtained only in the case where the two inputs (acoustic noise and ground motion) have been used. This confirms that vibrations of the cantilever in this condition were due to the two noise sources. Then, perturbations with acoustic origins can also be accurately considered in the model. The assumption of pinpoint forces acting on the discretized cantilever for modeling effects of acoustic noises is then sufficient when considering small vibrations of the cantilever (nanometer tip vibrations of a millimeter sized cantilever). 
Note that, in this study, we have considered only the perpendicular component of the wave. This is because a cantilever has a minimum stiffness in this direction and in this case acoustic noises have the most effect on the tip deflection. In real life, acoustic noises can come from many directions but it is possible to consider the component of the noise which has the most effect on the cantilever.

In (Fig. 22) and (Fig. 24), the level of the r.m.s. vibrations around the resonance are close to each other such as explained in section 4.2 (see table 2 for conditions 1 and 2). However, behaviors of the PSDs are completely different because the sources of noise are not the same. This demonstrates the interest of describing accurately origins of noises for the control part.

In this study, we have considered the vibrations of the cantilever only at the first resonance mode although the model is able to describe other resonances. Indeed, we could observe through experimental measurements that in all studied environmental conditions, this mode was always predominant.

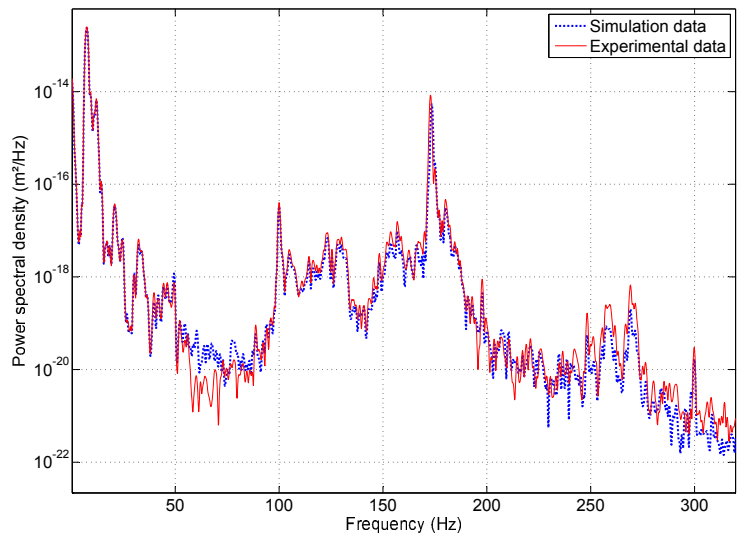

Fig. 22. Power spectral density of the cantilever 1 tip vibrations subject to ground motion (condition 1).

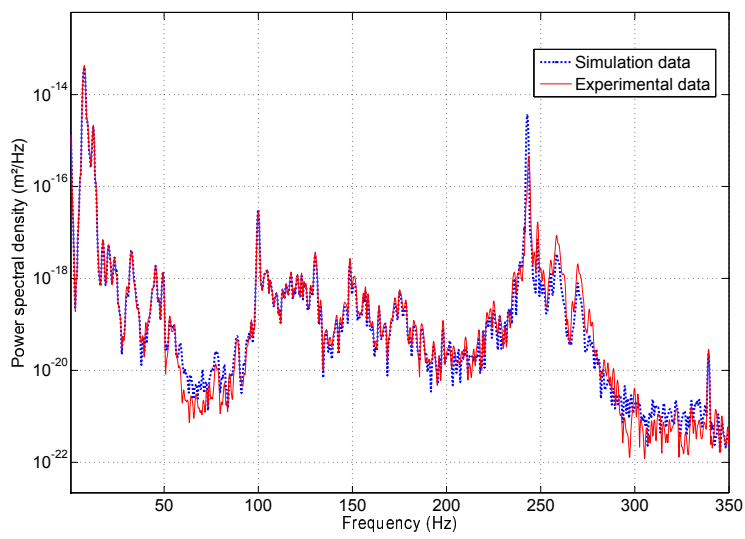

Fig. 23. Power spectral density of the cantilever 2 tip vibrations subject to ground motion (condition 1).

Moreover, the model presented in this paper contains 1024 states. As a consequence, the dynamic response computation can be long. An order reduction is then needed and especially when using the model in a feedback control. Various methods can be used to this end, such as the one using balancing techniques to compute reduced- order approximations [34]. The objective of the model reduction is to preserve only the predominant modes of the system and to reduce the computation time during simulations. In the case of the cantilever 1, 1024 states have been used for the initial model, but it can be reduced in the modal basis to capture accurately only dominant modes whose frequencies are less than $1 \mathrm{KHz}$. This allows obtaining a reduced order model which can be used in a feedback control.

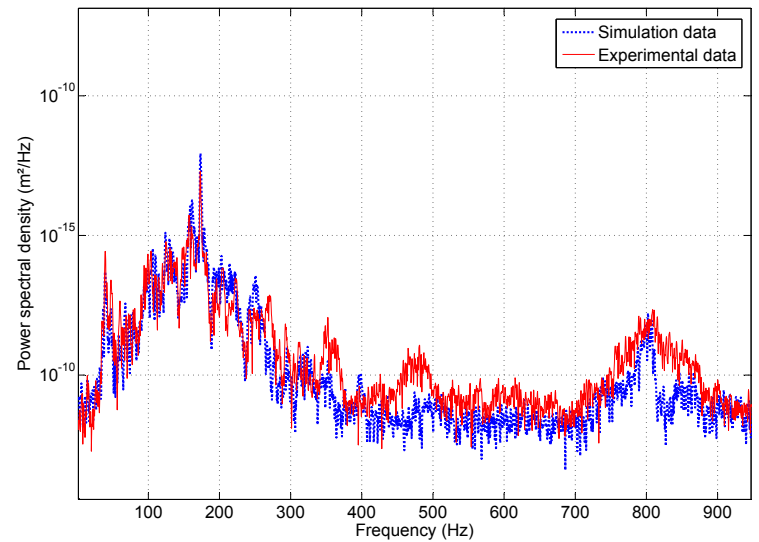

Fig. 24. Power spectral density of the cantilever 1 tip vibrations subject to acoustic noise $(45 \mathrm{~dB})$ (condition 2).

The practical long term application of the work reported in this paper is to improve with appropriate control strategies the closed loop performances of micromanipulation systems despite many sources of noise in a given environment. For instance, the dexterous manipulation of fragile samples (e.g. biological cells) requires gripping force control. The effectiveness of such operation can be improved if the noise is managed. In a modeling point of view, ground motion can be taken into account at each clamped part of the micromechanical structure and acoustic noises can be considered as acting along the end effectors of the microgripper.

\section{CONCLUSION}

In this paper, the sources of noise and vibrations in millimeter sized micromanipulation systems are investigated. Environmental noise including ground motion and acoustic noises has been characterized using a specific instrumentation. Effects of ground motion and acoustic noises have been evaluated on an experimental setup including cantilevers of different lengths designed with the respect of dynamical properties of millimeter sized micromanipulation systems commonly found in the literature. The purpose has been to identify, in different environmental conditions, noise sources from vibration measurements at the tip of a cantilever. To this end, a precise cantilever model is used to reconstruct the noise source from experimental tip measurements. The model is developed in the state space allowing easy simulations and uses for the control through the Matlab/Simulink software. Ground motion has been evaluated in a typical micromanipulation room and taking into account in the model as a base excitation. The model proved its efficiency to describe such perturbation. Experiments performed in an anechoic chamber 
demonstrated the effectiveness of the model to describe also tip vibrations of a cantilever due to acoustic noises. Then, in a given environment, the contribution of each source of noise on the vibration of systems intended for micromanipulation tasks can be evaluated, predicted, and noises can be rejected with an appropriate control strategy.

Future works will concern the use of active noise controllers to reduce effects of the environmental noise on positioning accuracy of millimeter sized micromanipulation systems. Then control strategies will depend on the environmental conditions (level of ground motion and acoustic noises). Moreover a characterization of noises on micrometric cantilevers such as used in atomic force microscopy will be investigated. In such systems, thermal noise must be taken into account in dynamic models as an internal perturbation and effects of environmental noise must be evaluated. Then, models allowing both the description of external (environment) and internal (thermal fluctuations) perturbations will be investigated for a large kind of micromanipulation systems whatever their size.

The authors would like to thank GUIBERT David for cantilevers fabrication.

\section{REFERENCES}

[1] Zubir M. N. M., Shirinzadeh B., "Development of a CompliantBased Microgripper for Microassembly", IEEE MESA 2008, Beijing, China.

[2] Chu D. T., Creemer J. F., Sarro P. M., "Lateral nano-Newton force-sensing piezoresistive cantilever for microparticle handling", J. Micromech. Microeng, vol. 16, number. 6, pp. 102 - 106, 2006

[3] Eisinberg A., Tonet O., Dario P., Macri G., Carrozza M. C., "Microfabricated Instruments for Fetal Cardiac Surgery: Experiments on Haptic Tissue Recognition", International Symposium on Haptic Interfaces for Virtual Environment and Teleoperator Systems, Virginia, USA, 2006.

[4] Beyeler F., Neild A. P., Oberti S., Bell D. J., Sun Y., Dual J., Nelson B. J., "Monolithically Fabricated Micro-Gripper with Integrated Force Sensor for Manipulating Micro-Objects and Biological Cells Aligned in an Ultrasonic Field", IEEE/ASME Journal of Microelectromechanical Systems (JMEMS), vol. 16, number. 1, pp. 7 - 15, 2006.

[5] Xinyu L., Jianhua T., Yu S., "A millimeter-sized nanomanipulator with sub-nanometer positioning resolution and large force output", Smart Materials and Structures, vol. 16, number. 5, pp. $1742-1750,2007$.

[6] Zubir M. N. M., Shirinzadeh B., "Development of a high precision flexure-based microgripper", Precision Engineering, vol. 33, issue. 4, pp. 362-370, 2009.

[7] Haddab Y., Chen Q., Lutz P., "Improvement of strain gauges micro-forces measurement using Kalman optimal filtering", Mechatronics, vol. 19, issue. 4, pp. 457-462, 2009

[8] Jain R. K., Patkar U. S., Majumdar S., "Micro gripper for micromanipulation using IPMCs (ionic polymer metal composites)", Journal of Scientific and Industrial Research, vol. 68, pp 23-28, 2009.

[9] Anis Y. H., Cleghorn W. L., Mills J. K., "Modal Analysis of Microgrippers used in Assembly of MEMS Devices", International Conference on MEMS,NANO and Smart Systems, Banff, Alberta, Canada , 2005.

[10] Rakotondrabe M., Clevy C., Lutz P., "Complete Open Loop Control of Hysteretic, Creeped, and Oscillating Piezoelectric Cantilevers ", IEEE Transactions on Automation Science and Engineering, vol. 7, issue. 3, pp. 440-450, 2009.

[11] Martinez J., Panepucci R., "Design, Fabrication, and Characterization of a Microgripper Device" in Proceedings of the Florida Conference on Recent Advances in Robotics, 2007.

[12] Kim K., Liu X., Zhang Y., Sun Y., "MicroNewton ForceControlled Manipulation of Biomaterials Using a Monolithic MEMS Microgripper with Two-Axis Force Feedback" In Proc. of the IEEE International Conference on Robotics and Automation, Pasadena, CA, USA, 2008.
[13] Chen T., Chen L., Sun L., Wang J., Li X., "A Sidewall Piezoresistive Force Sensor Used in a MEMS Gripper " Lecture Notes in Computer Science, vol. 5315, pp. 207-216, 2008.

[14] Yang G., Gaines J. A., Nelson B. J. "A Flexible Experimental Workcell for Efficient and Reliable Wafer-Level 3D Microassembly" In Proc. of the IEEE International Conference on Robotics and Automation, Seoul, Korea, 2001.

[15] Paolino P., Tiribilli B., Bellon L., "Direct measurement of spatial modes of a microcantilever from thermal noise", Journal of Applied Physics, vol. 106, issue. 9, pp. 094313 - 094313-5 , 2009.

[16] Gittes F., Schmidt C. F., "Thermal noise limitations on micromechanical experiments ", European biophysics journal, vol. 27, pp. $75-81,1998$.

[17] Zhou Q., Aurelian A., Del C. C., Esteban P. J ., Kallio P., Bo C., Koivo H. N., "A microassembly station with controlled environment", Microrobotics and microassembly. vol. 4568, pp. 252-260, 2001.

[18] Brenan C. J. H., Charette P. G., Hunter I. W., "Environmental isolation platform for microrobot system development", Review of Scientific Instruments, vol. 63, issue. 6, pp. 3492 - 3498, 1992.

[19] Park J., Moon W. "The systematic design and fabrication of a three-chopstick microgripper" The International Journal of Advanced Manufacturing Technology, vol. 26, number. 3, pp. 251-261, 2005.

[20] Rakotondrabe M., Agnus J., Rabenorosoa K., Chaillet N., "Characterization, modeling and robust control of a nonlinear 2-dof piezocantilever for micromanipulation/microassembly", IEEE/RSJ - IROS, International Conference on Intelligent Robots and Systems, St Louis MO, USA, 2009.

[21] Tamadazte B., Lefort-Piat N., Dembl S., Fortier G., "Robotic micromanipulation for microassembly : modelling by sequencial function chart and achievement by multiple scale visual servoings.", Journal of micro-nano-mechatronics, vol. 5, pp. 1-14, 2009.

[22] Tamadazte B., Lefort-Piat N., Dembl S., "Robotic Micromanipulation and Microassembly Using Monoview and Multiscale Visual Servoing.", IEEE/ASME Transactions on Mechatronics, vol. 16, pp. 277-287, 2009.

[23] Grossard M. Boukallel M. Chaillet N. Rotinat-Libersa C., "Modeling and Robust Control Strategy for a ControlOptimized Piezoelectric Microgripper", IEEE/ASME Transactions on Mechatronics, vol. PP, issue: 99, pp. 1-10, 2010.

[24] Tang X., Chen I. M., "Robust control of XYZ flexure-based micromanipulator with large motion ", Frontiers of Mechanical Engineering in China, vol. 4, number: 1, pp. 25-34, 2009.

[25] Rakotondrabe M., Haddab Y., Lutz P., "Quadrilateral Modelling and Robust Control of a Nonlinear Piezoelectric Cantilever", IEEE TCST, vol. 17, no. 3, pp. 528-539, 2009.

[26] Bhat N., Kim W. J., "Precision force and position control of ionic polymermetal composite" Journal of Systems and Control Engineering, vol. 218, number. 6, pp. 421-432, 2004

[27] Butt H. J., Jaschke M., "Calculation of thermal noise in atomic force microscopy ", Nanotechnology, vol. 6, number. 1, 1995.

[28] Boudaoud M., Haddab Y., Le Gorrec Y. , "Modelling of a MEMS-based microgripper: application to dexterous micromanipulation", IEEE/RSJ IROS 2010, Taipei, Taiwan.

[29] Menciassi A., Scalari G., Eisinberg A., Anticoli C., Francabandiera P., Carrozza M C., Dario P., "An Instrumented Probe for Mechanical Characterization of Soft Tissues ", Biomedical Microdevices, vol. 3, number. 2, pp. 149-156, 2001.

[30] Rayleigh L., "Theory of Sound" Dover Publications, New-York, 1877.

[31] Chao A. W., Tigner M. "Handbook of accelerator physics and engineering". World Scientific Publishing Co. Pte. Ltd., Singapore, 1999. $650 \mathrm{p}$.

[32] Seryi A., Ruland R., Baklakov B., Chupyra A., Erokhin A., Kondaurov M., Medvedko A., Parkhomchuk V., Singatulin S., Shubin E., Lach J., Plant D., Shiltsev V., "Hydrostatic level system for slow ground motion studies at FERMILAB and SLA", Proceedings of the Particle Accelerator Conference, Chicago, USA, 2001

[33] Bolzon B.,Brunetti L.,Jeremie A., Tomassini S., Rotundo U., Esposito M., "Preliminary ground motion measurements at LNF site for the super B project", Proceedings of the International Particle Accelerator Conference, Kyoto, Japan, 2010.

[34] Varga A., "Balancing-Free Square-Root Algorithm for Computing Singular Perturbation Approximations", IEEE CDC, Brighton, UK, 1991. 